\title{
MR Imaging of Subcallosal Artery Infarct Causing Amnesia after Surgery for Anterior Communicating Artery Aneurysm
}

\author{
S. Mugikura, H. Kikuchi, T. Fujii, T. Murata, K. Takase, E. Mori, S. Marinković, and S. Takahashi
}

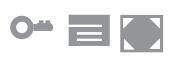

\begin{abstract}
BACKGROUND AND PURPOSE: During surgery to treat an aneurysm in the anterior communicating artery, injury to the subcallosal artery, a perforator of the anterior communicating artery, may lead to infarction that produces basal forebrain amnesia after surgery. Our purpose was to examine whether 3D MR imaging can detect subcallosal artery infarction in patients with amnesia after surgery for an anterior communicating artery aneurysm.
\end{abstract}

MATERIALS AND METHODS: We evaluated 3D-T2-weighted MR images obtained a median of 4 months after treatment of anterior communicating artery aneurysm for the presence of infarcted foci in 10 consecutive patients with postoperative amnesia. Because the subcallosal artery and its neighboring perforator, the recurrent artery of Heubner, were considered the most easily affected vessels during that surgery, we focused mainly on 8 regions of the subcallosal artery territory per hemisphere and 5 regions of the recurrent artery of Heubner territory per hemisphere.

RESULTS: All 10 patients had infarcts in the territory of the subcallosal artery (median, 9 regions per patient), and most were bilateral ( 9 of 10 patients). Five patients had additional infarcted foci in the territory of the recurrent artery of Heubner (median, 1 region per patient), all unilateral. Among the regions perfused by the subcallosal artery, the column of the fornix was involved in all patients; the anterior commissure, in 9; and the paraterminal gyrus, in 8 patients.

CONCLUSIONS: 3D MR imaging revealed subcallosal artery infarction, the distribution of which was mostly bilateral, presumably owing to the unpairedness of that artery, in patients with postoperative amnesia after anterior communicating artery aneurysm repair.

ABBREVIATIONS: $\mathrm{ACOA}=$ anterior communicating artery; $\mathrm{IQ}=$ full-scale intelligence quotient; $\mathrm{MQ}=$ general memory quotient; $\mathrm{RAH}=$ recurrent artery of Heubner; VISTA = volumetric isotropic turbo spin-echo acquisition

$S^{i}$ ince the 1950s, amnesia sufficient to affect quality of life has been repeatedly reported in patients following surgical repair of anterior communicating artery (ACoA) aneurysms. ${ }^{1-6}$ On the basis of descriptions from the 1970s of the perforating branches from the $\mathrm{ACoA},{ }^{7}$ much indirect evidence indicates that the amnesia is caused by damage or occlusion of the perforators of the

Received March 23, 2014; accepted after revision June 4.

From the Departments of Diagnostic Radiology (S. Mugikura, T.M., K.T., S.T.) and Behavioral Neurology and Cognitive Neuroscience (H.K., T.F., E.M.), Tohoku University, Graduate School of Medicine, Sendai, Japan; and Institute of Anatomy (S. Marinković), School of Medicine, University of Belgrade, Belgrade, Serbia.

Please address correspondence to Shunji Mugikura, MD, PhD, Department of Diagnostic Radiology, Tohoku University, Graduate School of Medicine, 1-1 Seiryomachi, Aoba-ku, Sendai, Japan 980-8574; e-mail: mugi@rad.med.tohoku.ac.jp

- Indicates open access to non-subscribers at www.ajnr.org

EIndicates article with supplemental on-line table.

Indicates article with supplemental on-line photo.

http://dx.doi.org/10.3174/ajnr.A4057
ACoA, and this is known as ACoA syndrome. ${ }^{2,8,9}$ Gade $^{10}$ linked vascular damage of the perforators from the ACoA during surgery with postoperative amnesia not directly, but clearly. In brief, the author found that postoperative amnesia occurred more frequently in cases of trapping the $\mathrm{ACoA}$ ( $82 \%$ of 11 patients) than in clipping the aneurysmal neck ( $16 \%$ of 37 patients). The author presumed that trapping the ACoA, which completely disrupted the blood supply through perforators from the ACoA, resulted in a high prevalence of postoperative amnesia. Another report from a postmortem examination of a patient with amnesia following a ruptured and repaired ACoA aneurysm revealed infarctions in the basal forebrain bilaterally, and the author concluded that infarction was caused by an inadvertent sacrifice of the perforators of the ACoA, which presumably was responsible for the patient's amnesia. ${ }^{11}$

Several authors have suspected that the amnesia is caused by occlusion or damage of the subcallosal artery, among the perforating arteries of the ACoA. ${ }^{12-15}$ The artery is the largest unpaired 

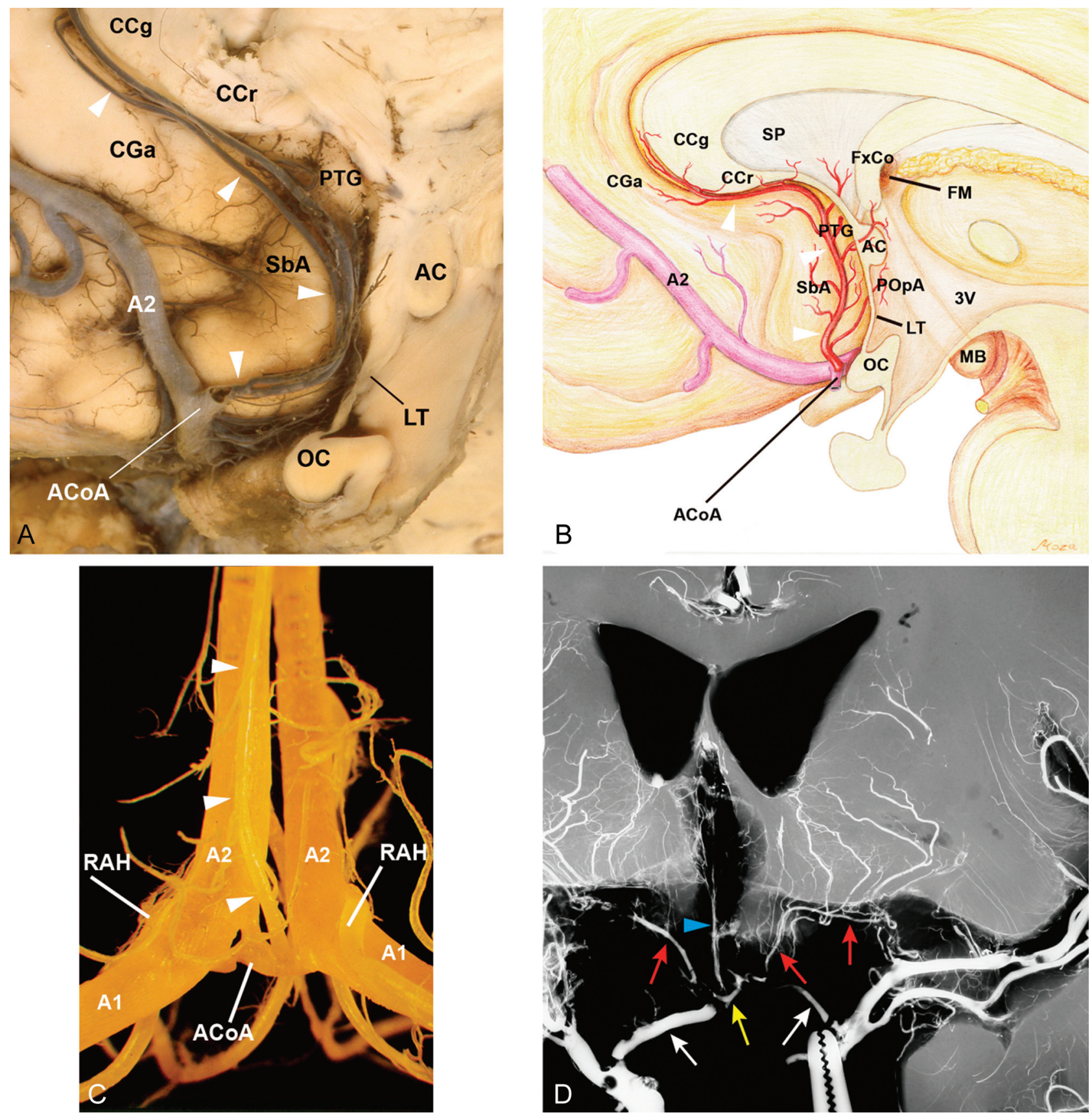

FIG 1. Anatomy of the subcallosal artery and RAH and their supplying basal forebrain regions. $A$, Specimen shows the subcallosal artery. The unpaired subcallosal artery (arrowheads) originates from the posterosuperior aspect of the ACoA, divides into 2 stems, and ascends dorsally into the lamina terminalis (LT) cistern, then curves upward and forward along the LT, paraterminal gyrus (PTG), subcallosal area (SbA), the rostrum $(\mathrm{CCr})$ and genu $(\mathrm{CCg})$ of the corpus callosum, and the anterior cingulate gyrus (CGa), thus exhibiting a characteristic S-shaped curve. OC indicates optic chiasm. B, Illustrative figure of the subcallosal artery (arrowheads) supplying the basal forebrain on the basis of our previous study regarding the microsurgical anatomy of the artery. ${ }^{16}$ The subcallosal artery originates from the posterosuperior aspect of the ACoA, ascends dorsally into the LT cistern, and supplies the 8 regions of the basal forebrain as follows: preoptic area (POpA), PTG including a part of the septum pellucidum (SP), SbA, anterior commissure (AC), and column of fornix ( $\mathrm{Fx} C \mathrm{Co}$ ), then curves forward and upward to supply the $\mathrm{CCr}$, $\mathrm{CCg}$, and $\mathrm{CGa}$. FM indicates foramen of Monro; MB, mammillary body; A2, A2 segment of the anterior cerebral artery; $3 \mathrm{~V}$, third ventricle. $C$, Specimen of the anterior cerebral and ACOA complex injected with methacrylic resin viewed from the posterior side. The subcallosal artery (arrowheads) is seen arising from the ACOA, A1, right and left A1 segments, and A2, right and left A2 segments, of the anterior cerebral arteries. D, Coronal microangiogram of the RAH on both sides and the unpaired subcallosal artery (reproduced, with permission, from Takahashi S, Goto K, Fukasawa $\mathrm{H}$, et al. Computed tomography of cerebral infarction along the distribution of the basal perforating arteries. Part I. Striate arterial group. Radiology 1985;155:107-18). Both internal carotid arteries have been retracted inferiorly to demonstrate the cisternal course of the RAH (red arrows), which follows a curved or tortuous course along the Al segment of the anterior cerebral artery (white arrows). The branches of the RAH are distributed to a part of the basal forebrain. The subcallosal artery (arrowhead) is also seen arising from the ACoA (yellow arrow).

one with a diameter of approximately $0.5 \mathrm{~mm}$ and perfuses the medial and ventral cerebral hemispheres (basal forebrain) bilaterally (Fig 1). ${ }^{13,16}$ The proximity between ACoA aneurysms and the origin of this artery suggests the possibility of arterial injury during surgery (On-line Fig 1).

In a review of 17 neurosurgical outcome studies (including 
Table 1: Patient demographic data and summary of neuropsychological findings

\begin{tabular}{|c|c|c|c|c|c|c|c|c|c|c|}
\hline Patient & 1 & 2 & 3 & 4 & 5 & 6 & 7 & 8 & 9 & 10 \\
\hline Age (yr) (sex) & $52 / \mathrm{M}$ & $42 / M$ & $39 / \mathrm{M}$ & $45 / M$ & $54 / M$ & $45 / M$ & $69 / M$ & $55 / M$ & $39 / M$ & $59 / F$ \\
\hline Ruptured/unruptured & $\mathrm{R}$ & $\mathrm{R}$ & $\mathrm{R}$ & $\mathrm{R}$ & $\mathrm{R}$ & $\mathrm{R}$ & U & $\mathrm{R}$ & $\mathrm{R}$ & $U$ \\
\hline$C T$ grade $^{a}$ & 3 & 4 & 3 & 3 & 4 & 3 & NA & 4 & 4 & NA \\
\hline Treatment & Trap & Clip & Clip 2nd & Clip & Clip & Clip & Clip & Clip & Clip & Clip \\
\hline Months from treatment ${ }^{\mathrm{b}}$ & 2 & 4 & $4^{\mathrm{b}}$ & 3 & 13 & 3 & 3 & 5 & 3 & 4 \\
\hline MMSE $^{\mathrm{C}}$ & 24 & 25 & 26 & 28 & 25 & 28 & 26 & 24 & 23 & 27 \\
\hline $\mathrm{IQ}^{\mathrm{d}}$ & 82 & 97 & 111 & 110 & 120 & 106 & 102 & 83 & 89 & 92 \\
\hline$M Q^{e}$ & 58 & 59 & 68 & 92 & 92 & 87 & 86 & 64 & 68 & 67 \\
\hline IQ minus $M Q$ & 24 & 38 & 43 & 18 & 28 & 19 & 16 & 19 & 21 & 25 \\
\hline Attention/concentration & 81 & 80 & 114 & 138 & 131 & 115 & 115 & 91 & 94 & 133 \\
\hline Delayed recall & $<50$ & $<50$ & $<50$ & $<50$ & 64 & 73 & 62 & $<50$ & $<50$ & $<50$ \\
\hline
\end{tabular}

Note:- R indicates ruptured; U, unruptured; MMSE, Mini-Mental State Examination; IVH, intraventricular hemorrhage in the bilateral lateral ventricles; Trap, trapping; Clip, clipping; NA, not applied; WAIS-III, Wechsler Adult Intelligence Scale III; WMS-R, Wechsler Memory Scale-Revised.

${ }^{a} \mathrm{CT}$ grade, proposed by Claassen et al, ${ }^{39}$ classifies the severity of aneurysmal subarachnoid hemorrhage on CT scans at onset into 5 grades from 0 to 4 , according to the appearance of both of SAH and intraventricular hemorrhage in the bilateral lateral ventricles: grade 0, no SAH or IVH; grade 1, minimal SAH, no IVH; grade 2, minimal SAH, with IVH; grade 3, thick SAH, no IVH; grade 4, thick SAH, with IVH. In this scaling, the definition of "thick" is "completely filling" $\geq 1$ cistern or fissure. In 2 patients with unruptured aneurysm (patients 7 and 10), grading was not applied.

${ }^{\mathrm{b}}$ Months after second clipping.

${ }^{c}$ MMSE, used to assess cognitive impairment (full score 30). ${ }^{40}$

${ }^{d}$ IQ evaluated by the Wechsler Adult Intelligence Scale III. ${ }^{18}$

${ }^{\mathrm{e}} \mathrm{MQ}$, general memory quotient, attention/concentration quotient, and delayed recall quotient, evaluated by Wechsler Memory Scale-Revised. ${ }^{19}$ Each quotient has a mean of 100 in the normal population and an SD of 15. A substantial difference between IQ (by the WAIS-III) and MQ (by the WMS-R) scores indicates that the person with amnesia has a particular impairment in memory_but not in the "intelligence" per se. ${ }^{20}$

504 patients) of ruptured and repaired ACoA aneurysms, the diagnosis of basal forebrain amnesia has been made mainly by neuropsychological examination alone, thereby leading to a large variety of prevalences ranging between 3\% and 83\%. ${ }^{17}$ Meanwhile, in the reports that provided imaging findings in cases with postoperative amnesia of an ACoA aneurysm, most used CT scans and/or 2D MR imaging (with relatively thick section thickness) and did not clarify the exact location of the infarcts inside the basal forebrain, probably because of low image resolution and metallic artifacts from clips placed during surgery. ${ }^{2,3,5}$

Our purpose was to test the hypothesis that 3D MR imaging can detect subcallosal artery infarction in patients with amnesia after ACoA aneurysm surgery.

\section{MATERIALS AND METHODS \\ Patients}

Our institutional review board approved this retrospective observational study, and written informed consent was waived. From December 2007 to March 2013, 14 consecutive patients with amnesia following surgical treatment of an ACoA aneurysm visited the behavioral neurology service of our hospital and underwent 3T 3D MR imaging examinations. Patients were examined by behavioral neurologists and underwent neuropsychological examinations, including the Wechsler Adult Intelligence Scale III $^{18}$ and Wechsler Memory Scale-Revised. ${ }^{19}$ Of the 14 patients, we enrolled 10 patients who showed a substantial difference between the fullscale intelligence quotient (IQ) on the Wechsler Adult Intelligence Scale III and general memory quotient (MQ) on the Wechsler Memory Scale-Revised scores; IQ - MQ > 15, indicating that the person with amnesia had a particular impairment in memory but not in "intelligence" per $\mathrm{se}^{20}[\mathrm{IQ}-\mathrm{MQ}=26$ (mean), 18-43 (range), all $>15$ ]. Patient demographic data and a summary of neuropsychological findings are shown in Table 1. The 10 patients included 8 with ruptured and 2 with unruptured ACoA aneurysms.

Neuropsychological examinations and 3D MR images reviewed in this study were performed a median of 4 months (range, 2-13 months) from aneurysm treatment. Each set of examinations was conducted within a month of the other. This delayed timing of examinations was considered desirable for neuropsychological memory assessment because such assessment in the acute phase (from 1 to 6 weeks) after the aneurysmal rupture would hardly be valid, with the diagnosis of amnesia being difficult due to both a state of confusion, including disorientation, and intellectual disturbance. ${ }^{17}$ Indeed, amnesia did not manifest for some time in 7 patients after treatment of the ruptured ACoA aneurysms (surgical clipping in 6 patients and trapping of the ACoA in another); however, amnesia became apparent as the patients recovered from acute-stage illness. In contrast, 2 patients with unruptured ACoA aneurysms (patients 7 and 10) developed amnesia immediately after surgery. The tenth patient (patient 3 ) underwent clipping of an ACoA aneurysm the day following the rupture and was doing well when approximately 3 weeks later, postoperative CT angiography disclosed a remnant of the aneurysm lumen. After a second surgery, the patient immediately presented with amnesia.

\section{MR Imaging Examination}

In all 10 patients, $M R$ imaging examinations were performed a median of 4 months (range, 2-13 months) from aneurysmal treatment by using a 3T machine (Achieva 3T Quasar Dual imager; Philips Healthcare, Erlangen, Germany) with an 8-channel sensitivity encoding head coil. All patients underwent a sagittal $3 \mathrm{D}$ volumetric isotropic turbo spin-echo acquisition (VISTA) of T2-weighted imaging, axial 3D magnetization prepared rapid acquisition of gradient-echo, conventional axial T1-, T2-, and T2* weighted, fluid-attenuated inversion recovery imaging, and timeof-flight MR angiography. VISTA provides high-resolution volumetric T2-weighted images with a turbo spin-echo acquisition and produces fewer metallic artifacts from aneurysmal clips than the fast-field echo acquisition.

The parameters for T2WI-VISTA were the following: TR/TE, 4000/180 ms; FOV, foot to head, $224 \mathrm{~mm}$, anterior to posterior, 
Table 2: Infarction in each vascular territory on MR imaging for 10 patients ${ }^{\mathrm{a}}$

\begin{tabular}{|c|c|c|c|c|c|c|c|c|c|c|c|c|c|c|c|c|c|c|c|c|}
\hline & \multicolumn{20}{|c|}{ Patient No. (R/L) } \\
\hline & \multicolumn{2}{|c|}{1} & \multicolumn{2}{|c|}{2} & \multicolumn{2}{|c|}{3} & \multicolumn{2}{|c|}{4} & \multicolumn{2}{|c|}{5} & \multicolumn{2}{|c|}{6} & \multicolumn{2}{|c|}{7} & \multicolumn{2}{|c|}{8} & \multicolumn{2}{|c|}{9} & \multicolumn{2}{|c|}{10} \\
\hline & $\mathbf{R}$ & $\mathbf{L}$ & $\mathbf{R}$ & $\mathbf{L}$ & $\mathbf{R}$ & $\overline{\mathrm{L}}$ & $\mathbf{R}$ & $\overline{\mathrm{L}}$ & $\mathbf{R}$ & $\overline{\mathrm{L}}$ & $\mathbf{R}$ & $\overline{\mathrm{L}}$ & $\mathbf{R}$ & $\overline{\mathrm{L}}$ & $\mathbf{R}$ & $\overline{\mathrm{L}}$ & $\mathbf{R}$ & $\overline{\mathrm{L}}$ & $\mathbf{R}$ & L \\
\hline \multicolumn{21}{|l|}{ Vascular territory } \\
\hline Subcallosal $(n=8)$ & + & + & + & + & + & + & + & + & + & + & + & + & & + & + & + & + & + & + & + \\
\hline $\operatorname{RAH}(n=5)$ & + & & & & & & & & + & & & & & & & + & + & & + & \\
\hline Unspecified $(n=3)$ & + & & & & & + & & & & & + & & & & + & + & + & + & + & \\
\hline
\end{tabular}

Note:- R/L indicates right and left hemisphere; +, infarcted focus present in a vascular territory; blank space, sparing of the territory.

a For vascular territories of subcallosal, RAH, and unspecified, refer to Fig. 2.

$224 \mathrm{~mm}$, right-to-left, $164 \mathrm{~mm}$; acquisition voxel size, foot to head, $0.56 \mathrm{~mm}$, anterior to posterior, $0.56 \mathrm{~mm}$, right-to-left, 0.80 $\mathrm{mm}$; reconstruction voxel size, foot to head, $0.44 \mathrm{~mm}$, anterior to posterior, $0.44 \mathrm{~mm}$, right-to-left, $0.40 \mathrm{~mm}$; echo-train length, 111; number of signal averages, 1 . VISTA uses the low-refocusing flip angle scheme to reduce initial signal modulations during the first few echoes by setting the flip angle at $130^{\circ}$ for the first refocusing pulse and $80^{\circ}$ for succeeding refocusing pulse trains. ${ }^{21} \mathrm{We}$ used sensitivity encoding or parallel imaging with a speed-up factor of 6.0 ( 2 in the anterior to posterior, 3 in the right-to-left direction). Total acquisition time was 8 minutes 16 seconds. We obtained T2WI-VISTA images in the sagittal plane for acquisition within a clinically reasonable scanning time.

The parameters for MPRAGE were the following: TR/TE, 6.6/ $3.0 \mathrm{~ms}$; FOV, foot to head, $176 \mathrm{~mm}$, anterior to posterior, 224 $\mathrm{mm}$, right-to-left, $204 \mathrm{~mm}$; acquisition voxel size, 1/1/1 mm; reconstruction voxel size, $0.5 / 0.5 / 0.5 \mathrm{~mm}$; echo-train length, 226 ; number of signal averages, 1 ; flip angle, $8^{\circ}$. The total scan duration was 5 minutes 13 seconds.

In addition to the $3 \mathrm{D}$ images obtained in the chronic phase as described, 3 patients underwent MR imaging in the acute stage after treatment at outside hospitals by using $1.5 \mathrm{~T}$ machines (Signa; GE Healthcare, Milwaukee, Wisconsin). The images were sent to us later for review: MR imaging including diffusionweighted imaging immediately after surgery in 2 patients with unruptured ACoA aneurysms and MR imaging 1 week after a second surgery in another patient.

\section{Evaluation of MR Imaging Findings}

On multiplanar reconstruction images, 2 experienced neuroradiologists blinded to neuropsychological assessments, with 25 (S.T.) and 15 (S. Mugikura) years' experience, identified and localized infarctions by consensus.

Basal forebrain anatomy was identified on MPRs such as in On-line Fig 2, by referencing multiple coronal brain specimens with detailed diagrams (with 0.7 - to $1.5-\mathrm{mm}$ intersectional gap) by Mai et al. ${ }^{22}$ Regarding the vascular territories of infarction, we referenced descriptions of arterial supply of the basal forebrain in the radio-anatomic literature $e^{16,23,24}$ and specifically focused on the following 16 regions of the basal forebrain per hemisphere: 8 regions of the territory of the subcallosal artery (anterior cingulate gyrus; anterior commissure; column of the fornix; paraterminal gyrus, including a part of the septum pellucidum; preoptic area; rostrum and genu of the corpus callosum; and subcallosal area). ${ }^{13,16}$ Because the recurrent artery of Heubner (RAH), which usually originates on both sides around the junction of the ACoA and the anterior cerebral artery (Fig
1), ${ }^{25,26}$ is also considered at high risk of injury from treatment of ACoA aneurysms, 5 regions of the territory of the RAH were also included in this analysis (anterior limb of the internal capsule, caudate nucleus, globus pallidus, nucleus accumbens, and putamen). ${ }^{24,26,27}$ Three additional neighboring regions with unspecified vascular supply (bed nucleus of the stria terminalis, diagonal band of Broca, and substantia innominata) were reviewed for infarction.

Excluding 4 regions completely obscured by artifacts from aneurysmal clips, we examined 316 unobscured or nearly unobscured regions. As well, we recorded infarctions outside the above 16 regions in the basal forebrain.

We also examined which part of the column of the fornix the infarction affected (ie, the pars libera, including both post- and precommissural fibers, and the pars tecta just posterior to the anterior commissure [On-line Fig 3]).

Atrophy of the mammillary body is known to correlate positively with severity of damage to the ipsilateral column of the fornix from Wallerian degeneration. ${ }^{28}$ Therefore, 2 other neuroradiologists, with 15 (T.M.) and 10 (K.T.) years' experience and blinded to the purpose of the evaluation, also examined the mammillary bodies for possible atrophy and its relationship to the presence or absence of infarctions in the column of the fornix.

\section{RESULTS}

All 10 patients had infarcted foci in the territory of the subcallosal artery, and 9 patients had bilateral foci (Table 2). In contrast, only 5 patients had infarcted foci in the territory of the RAH, all unilateral (patients 1, 5, 8, 9, and 10). Infarcted foci in the territories of unspecified arteries were found in 6 patients. Foci of 3 patients (patients 2, 4, and 7) were limited to the territory of the subcallosal artery and did not involve the territories of the RAH or unspecified arteries. No patients had infarcted foci in the territory of the RAH alone without foci in the territory of the subcallosal artery.

In 2 patients with unruptured aneurysms, DWI performed the day after the surgery showed acute infarcted foci in the basal forebrain. The foci were later confirmed by the $3 \mathrm{D}$ MR imaging to involve the unilateral territory of the subcallosal artery but not of the RAH in 1 patient (patient 7) and the bilateral territories of the subcallosal artery and the unilateral RAH territory in the other (patient 10). In another patient with a second clipping surgery, thin-section CT preoperatively showed no lesions in the basal forebrain but DWI performed 1 week after a second surgery showed acute infarcted foci in the basal forebrain. 3D MR imaging 


\section{Number of hemispheres}

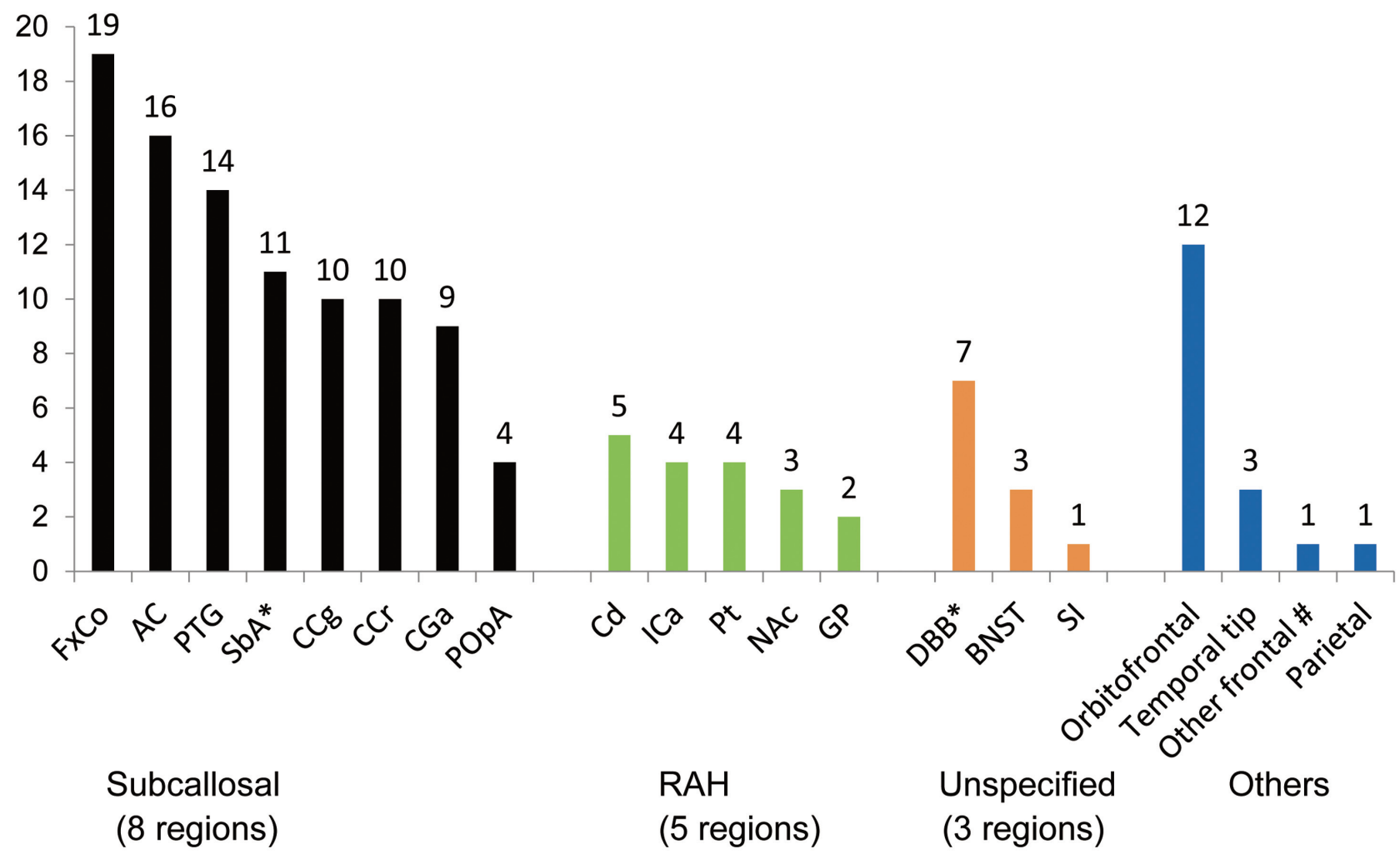

FIG 2. Summary of the infarcted foci on MR images in each region according to the vascular supply. The number on each bar graph represents the number of hemispheres in which infarcted foci of each region were found. Eight regions of the subcallosal artery: column of the fornix (FxCo), anterior commissure (AC), paraterminal gyrus (PTG), subcallosal area (SbA), genu of the corpus callosum (CCg), rostrum of the corpus callosum (CCr), anterior cingulate gyrus (CGa), and preoptic area (POpA). Five regions of the RAH: caudate nucleus (Cd), anterior limb of the internal capsule (ICa), putamen (Pt), nucleus accumbens (NAC), and globus pallidus (GP). Three other regions defined as the regions of unspecified vascular supply: diagonal band of Broca (DBB), bed nucleus of the stria terminalis (BNST), and substantia innominata (SI). The asterisk indicates that metallic artifacts from aneurysmal clips completely obscured the SbA in 2 hemispheres of 2 patients unilaterally and DBB in 1 patient bilaterally. Number sign indicates that "other frontal" represents the frontal lobe other than the orbitofrontal and basal forebrain region.

later disclosed bilateral infarcts in the territory of the subcallosal artery (patient 3).

Infarctions were most common in 3 regions in the territory of the subcallosal artery (Fig 2) - the column of the fornix, anterior commissure, and paraterminal gyrus (detailed MR imaging findings of 10 patients are in the On-line Table). The column of the fornix was involved in all 10 patients ( 19 hemispheres, bilaterally in 9 patients, unilaterally on the left in 1 patient). In the 19 hemispheres with fornix involvement, the pars libera was affected in all, while the pars tecta was affected in 15 (79\% of 19) hemispheres.

Bilateral involvement of the anterior commissure in 7 patients demonstrated a characteristic bow-tie-like appearance (Figs 3 and 4); unilateral involvement in 2 patients showed an incomplete bow-tie-like appearance (On-line Fig 4). All 7 patients with bowtie-like involvement of the bilateral anterior commissure had infarcts bilaterally in the adjoining pars libera of the column of the fornix, with associated infarcts in the pars tecta that were bilateral in 5 and unilateral in 2. Two patients had a lesion of the anterior commissure on only the left side, which therefore displayed an incomplete bow-tie-like appearance: They also had infarcted foci in the pars libera and pars tecta unilaterally on the left in 1 patient (patient 7, On-line Fig 4) and bilaterally in the other patient (patient 5). One patient had no involvement of the anterior commissure.

The paraterminal gyrus was involved bilaterally in 6 patients and unilaterally in 2, and the subcallosal area was involved bilaterally in 3 and unilaterally in 5 patients.

The corpus callosum was involved in the genu bilaterally in 5 patients and in the rostrum bilaterally in 3 and unilaterally in 4 patients. The anterior cingulate gyrus was involved bilaterally in 2 and unilaterally in 5 patients. These infarcted foci were located along the medial aspect of the brain and almost in line with associated lesions in the paraterminal gyrus and column of the fornix. This anteroposterior extent of involvements was observed on axial and sagittal MR images (Fig 4).

Of 3 regions with the unspecified vascular supply, the diagonal band of Broca was most frequently involved (35\%, 7 of 20 hemispheres; $50 \%, 5$ of 10 patients). Of regions outside the basal forebrain, the orbitofrontal region was most frequently involved (60\%, 12 of 20 hemispheres; $90 \%, 9$ of 10 patients).

Definite atrophy of the mammillary body was seen in $15(79 \%)$ of 19 hemispheres with infarcted regions in the column of the fornix. We considered the mammillary body to be nonatrophied 

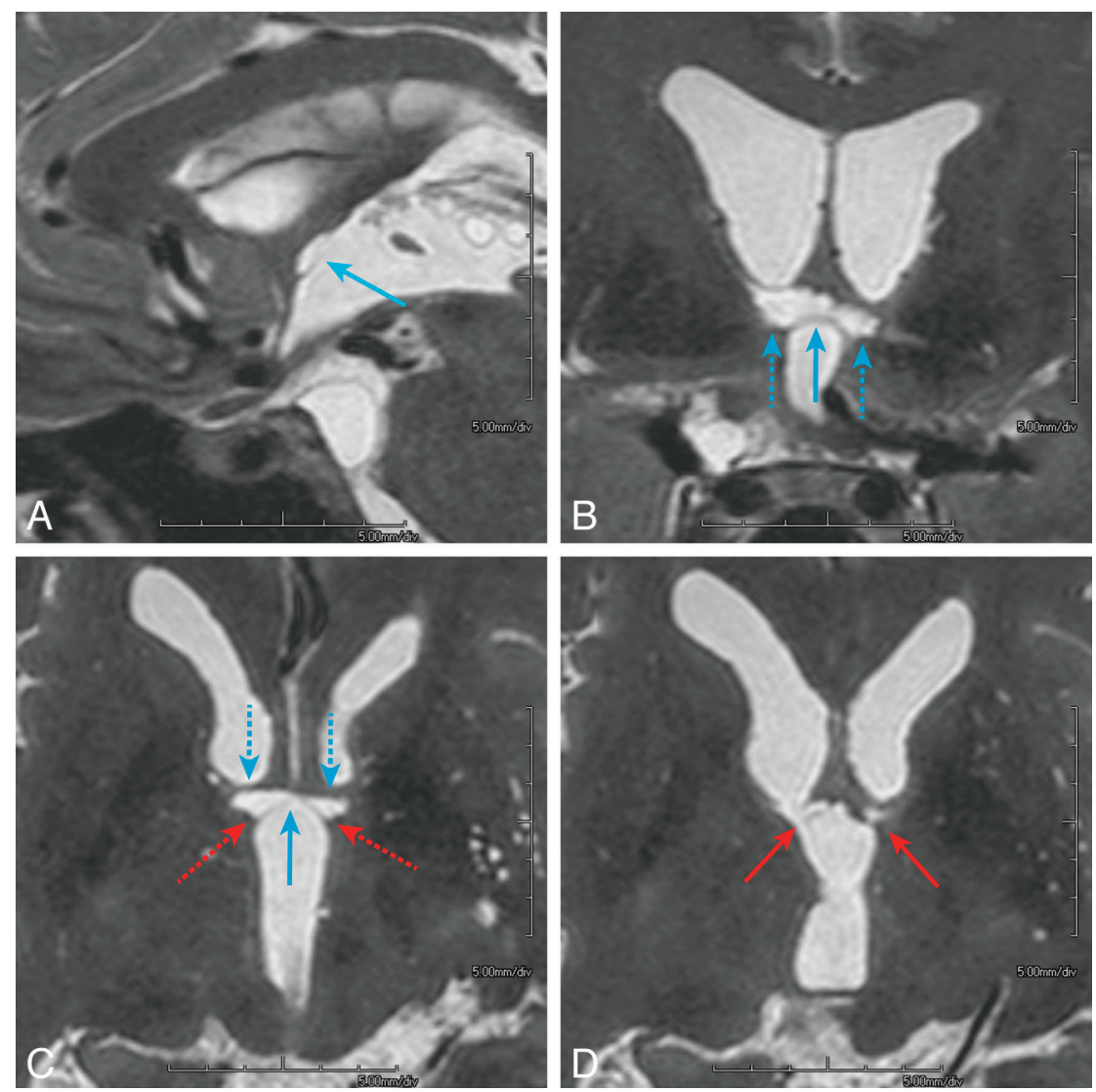

FIG 3. A 45-year-old who man presented with a ruptured aneurysm of the anterior communicating artery. Surgical clipping of the aneurysm was performed the day of onset (patient 4). Neuropsychological examination 3 months after the rupture confirmed amnesia. The imaging anatomy of the basal forebrain is detailed in On-line Fig 2: paramedian sagittal $(A)$, coronal $(B)$, and axial $(C)$ and its next superior section $(D)$, volumetric isotropic turbo spin-echo acquisition of T2WI (T2WI-VISTA) images shows infarcted foci in the midline (light blue arrows) and paramedian parts (dashed light blue arrows) of the anterior commissure along with the pars libera (red arrows) and pars tecta (dashed red arrows, $C$ ) of the columns of the fornices. Note that on coronal $(B)$ and axial $(C)$ images, infarcted foci in the bilateral anterior commissure show a characteristic bow-tie-like appearance and are associated with the infarcted foci in the adjoining bilateral pars libera and pars tecta of the column of the fornix. Other than the columns of the fornix and anterior commissure, no other regions are involved in the basal forebrain. The orbitofrontal region and temporal tip on the left were also involved, presumably damaged by the surgical procedure of the aneurysmal clipping (not shown).

in 2 hemispheres of 2 patients (patients 2 and 4) in which the mammillary body was compressed or deformed by the elongated posterior cerebral artery or the basilar artery. In a patient with unilateral fornix involvement by infarction in the left hemisphere, atrophy of the mammillary body was seen only in the left hemisphere (patient 7).

\section{DISCUSSION}

\section{Subcallosal Artery Infarct Causing ACoA Syndrome or Amnesia}

We found infarcted foci in the territory of the subcallosal artery in all 10 patients with amnesia following treatment of ACoA aneurysms, and in 9 patients, the infarcted foci were bilateral. Meanwhile, 5 patients had additional infarcted foci in the territory of the RAH, and its involvement was always unilateral. This difference between bilateral and unilateral involvement may be explained by the fact that the subcallosal artery is unpaired, whereas the RAH is present on both sides. Thus, infarct patterns in the cases described in this study are compatible with the suggestion of previous neurosurgical and neuropsychological studies that occlusion of the subcallosal artery could cause postoperative amnesia.

We believe that the relationship between surgical damage of the subcallosal artery and amnesia is particularly apparent in 3 patients who presented with amnesia immediately after surgery. In 2 patients with unruptured aneurysms and another with a second clipping surgery, DWI revealed acute lesions that were confirmed later by 3D MR imaging to involve the territory of the subcallosal artery. In all, the involvement in the basal forebrain was obviously caused by vascular damage during surgery.

This was a small study and may not be considered definitive to conclude that occlusion or damage of the subcallosal artery during surgery causes bilaterally distributed infarction and produces postoperative amnesia. However, this hypothesis would be extremely difficult to prove, unless a correlation was found between preserving a given artery and lack of amnesia. We believe the data that we present in this study are strong and appear to answer, to a reasonable degree, an important clinical question that has persisted for several decades. The causal relationship is apparent if we consider acutely developed amnesia immediately after surgery with postsurgically developed infarcts in the territory of the subcallosal artery in our 3 patients and the presence of infarcted foci in the same vascular territory in all the remaining 7 patients with amnesia following treatment of ACoA aneurysms.

\section{Sites Responsible for Postoperative Amnesia}

What structures of the basal forebrain are responsible for postoperative basal forebrain amnesia? This question remains to be answered. Because our study included no control group without amnesia, we cannot draw a definitive conclusion. However, considering the affected regions in our cases, the column of the fornix, a constituent of the Papez neuronal circuit (On-line Fig 3), seems the most likely responsible site for the amnesia. The same hypothesis has been suggested in the literature: Infarction relatively limited to the bilateral columns of the fornices on DWI has been separately reported in 4 patients with spontaneous stroke and acute onset of amnesia, ${ }^{29-32}$ in a patient with amnesia following clipping, ${ }^{33}$ and in another following coiling of an unruptured ACoA aneurysm. ${ }^{34}$ 

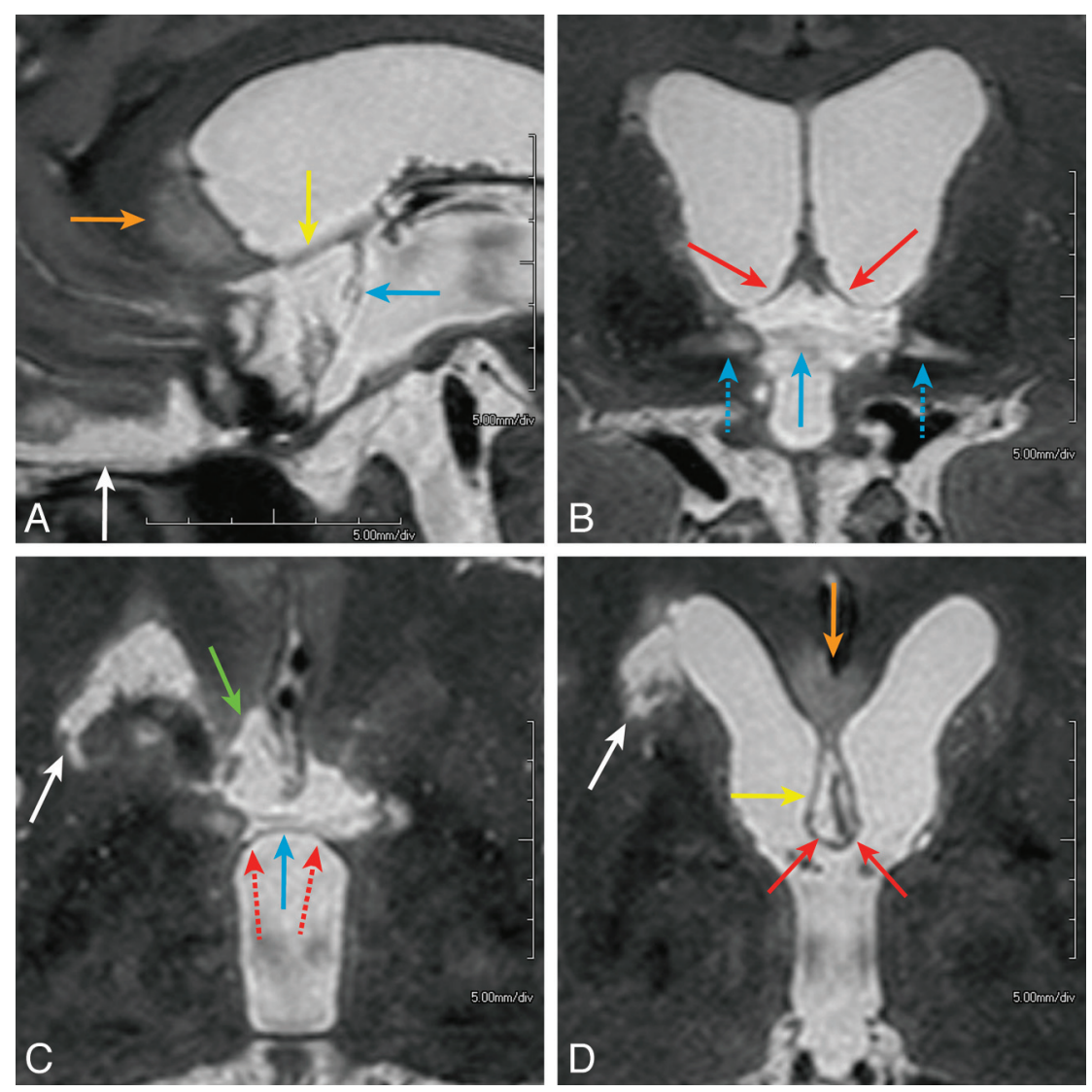

FIG 4. A 52-year-old man presented with a ruptured aneurysm of the anterior communicating artery. Surgical trapping of the ACoA for the ruptured ACoA aneurysm was performed the day of onset (patient 1). Neuropsychological examination 2 months after the rupture confirmed amnesia. Midsagittal $(A)$, coronal $(B)$, and axial $(C$ and $D)$ images of T2WI-VISTA show the infarcted foci involving the pars libera of the column of the fornix ( $B$ and $D$, solid red arrows) and anterior commissure ( $A-C$, light blue arrows), paraterminal gyrus ( $A$ and $D$, yellow arrows), subcallosal area ( $C$, green arrow), and genu or rostrum of the corpus callosum ( $A$ and $D$, orange arrows). The entire extent of the lesions shows sagittally elongated bandlike infarctions along the medial aspect of the brain, which probably represent the distribution of the characteristic $S$-shaped course of the subcallosal artery. On coronal $(B)$ and axial $(C)$ images, infarction in the bilateral anterior commissure shows a bow-tie-like appearance and is associated with infarcted foci in the adjoining pars libera (solid red arrows) and pars tecta (dashed red arrows) of the bilateral columns of the fornices. Lateral extension of hyperintense lesions along the lateral part of the anterior commissure ( $B$, dashed light blue arrows) may indicate Wallerian degeneration of the midline infarction in the anterior commissure (solid light blue arrow). Infarction in the head of the caudate nucleus on the right is also seen (white arrows, $C$ and $D$ ), which presumably represents involvement of the right recurrent artery of Heubner. The orbitofrontal region is also involved ( $A$, white arrow).

Furthermore, a recent study of diffusion tensor imaging in patients after treatment of a ruptured ACoA aneurysm also speculated that amnesia might be related to injury of the cingulum and the fornix. ${ }^{35}$ However, the authors did not analyze the sites of lesions or present the neuropsychological data of their cases. On the contrary, all our patients demonstrated infarction in the column of the fornix and underwent formal neuropsychological tests, which indicated their disproportionate impairment of memory but only limited intellectual decline [IQ - MQ $=26$ (mean), all $>15]$. We included only patients with relatively preserved intellectual function because diffuse brain damage owing to hydrocephalus and vasospasm could worsen generalized intellectual function with resultant memory impairment. Therefore, our manner of enrolling subjects may be a strength of our study because it enabled us to localize the lesions responsible for basal forebrain amnesia after ACoA aneurysmal treatment.

Still, we noted the high prevalence of infarctions in the orbitofrontal area in our study. The lesions in this area designated as infarction in this study could have included the sequelae of intraparenchymal hemorrhage associated with the aneurysmal rupture and/or partial surgical resection being necessary to visualize the ACoA aneurysmal neck during surgery. ${ }^{25}$ Several neuropsychological studies, however, indicated that the lesions in the orbitofrontal area alone did not cause amnesia. ${ }^{3,36,37}$

Alternatively, several reports attributed basal forebrain amnesia to interruption of the cholinergic system. ${ }^{1,3,11,38}$ Indeed, in our patients, infarction in the paraterminal gyrus in 8 and in the diagonal band of Broca in 5 suggests likely involvement of the cholinergic system. Additionally, lesions in the pars libera of the column of the fornix, which include cholinergic fibers, in all our patients might also indicate interruption of the cholinergic system.

\section{Diagnostic Value of 3D MR Imaging in Patients with Basal Forebrain Amnesia}

Because previous diagnosis of basal forebrain amnesia has been made mainly by the neuropsychological examination alone or by using CT and/or 2D MR imaging, detailed localization of lesions has not been achieved. In our study, in contrast, use of 3T 3D MR imaging enabled identification and localization of small infarcts, and we believe identifying the exact locations of infarcts in the basal forebrain should help clarify the diagnosis of post-treatment amnesia following surgery for ACoA aneurysms.

Specifically, 2 MR imaging signs appeared characteristic: The first, the bow-tie-like appearance of infarcts, seen on both axial and coronal MR images, represents bilateral involvement of the anterior commissure associated with infarcted foci in the column of the fornix (pars libera and pars tecta). The second sign, a sagittally elongated infarction along the medial aspect of the brain on axial or sagittal MR imaging planes that involves the anterior cingulate gyrus, genu, and/or rostrum of the corpus callosum, should represent the sagittally elongated vascular distribution along the characteristic S-shaped course of the subcallosal artery (Fig $1 A$, $-B$ ). We believe both signs are important $\mathrm{MR}$ imaging indicators of amnesia associated with ACoA aneurysm treatment.

AJNR Am J Neuroradiol 35:2293-301 Dec 2014 www.ajnr.org 
Although it may be possible to perform the same analysis by using conventional 2D 2- or 3-mm coronal imaging with/without axial images, we presume that 3D MR imaging by using MPR could more easily identify small structures in the basal forebrain. Indeed, a recent neuropsychological study by Proust et $\mathrm{al}^{5}$ revealed the high prevalence of verbal memory deficits $(58.3 \%)$ among 36 patients with variably decreased intellectual function after ACoA clipping. Regarding verbal memory deficits, Guglielmi ${ }^{14}$ stated, in his letter to the editor, that ACoA syndrome is likely due to occlusion of the subcallosal artery. In the series of reports by Proust et al using thin-section 1.5T 2D MR imaging (axial FLAIR images, 5-mm-thick; axial T2-weighted images, 2-mm-thick; axial T1-weighted images, 2.5-mm thick; and coronal T2-weighted images centered on the frontal and temporal areas, thickness not shown), the prevalence of the basal forebrain lesions was $33.3 \%$ in 36 patients. However, the authors did not clarify the exact location of lesions inside the basal forebrain. We believe that high-resolution 3D T2WI might be desirable for this kind of study.

\section{Limitations}

Our study limitations include a small number of patients and lack of a control group without symptoms of amnesia. Another limitation is that DWI was examined in the acute phase in 3 cases only, whereas in the remaining 7 patients with ruptured aneurysms, acute-phase DWI was not performed, presumably because the onset of amnesia after surgery was unnoticed or unrecognized due to associated acute illness. Further studies of a large number of consecutive patients treated surgically or interventionally for ACoA aneurysms, preferably by using both DWI in the acute phase and 3D MR imaging in the chronic phase along with formal neuropsychological examinations, remain to be conducted.

Another limitation is that we did not analyze the more detailed memory test, including retrieval or encoding. This analysis might clarify the relationship between the lesions and neuropsychological characteristics of postoperative amnesia.

The last limitation is the subjective evaluation by 2 examiners of atrophy of the mammillary bodies without volumetry.

\section{CONCLUSIONS}

We described 3D MR imaging findings in patients with amnesia following surgical treatment of ACoA aneurysms: infarctions in the territory of the subcallosal artery, mostly with bilateral involvement. In these cases, infarct patterns were compatible with previous neurosurgical and neuropsychological studies suggesting that occlusion of the subcallosal artery could cause postoperative amnesia. From a neurosurgical point of view, our results indicate that preservation of that artery during treatment of an ACoA aneurysm should be crucial, though further studies involving a large number of consecutive patients treated for ACoA aneurysms must be conducted to validate this hypothesis.

\section{ACKNOWLEDGMENTS}

We thank our department colleagues Yasuko Tatewaki and Li Li for their important suggestions and helpful discussions and Makoto Obara of Philips Electronics Japan, Tokyo, for technical advice and support in advanced MR imaging.
Disclosures: Shunji Mugikura-RELATED: Payment for Lectures (including service on Speaker Bureraus): Philips Healthcare, Terumo; UNRELATED: Grants/Grants Pending: Japan Society for the Promotion of Science Kakenhi*; Payment for Lectures (including service on Speakers Bureaus): Daiichi-Sankyo, Terumo, Philips Healthcare. Shoki Takahashi-UNRELATED: Grants/Grants Pending, JSPS Kakenhi, ${ }^{*}$ Daiichi-Sankyo,* Bayer,* Eisai,* Terumo,, Mitsubishi-Tanabe Pharma,* Nihon Medi-physics, ${ }^{\star}$ Philips Healthcare, ${ }^{*}$ Siemens, ${ }^{*}$ Toshiba, ${ }^{*}$ Payment for Lectures (including service on Speakers Bureaus): Daiichi-Sankyo, Eisai, Terumo. *Money paid to the institution.

\section{REFERENCES}

1. Damasio AR, Graffradford NR, Eslinger PJ, et al. Amnesia following basal forebrain lesions. Arch Neurol 1985;42:263-71

2. DeLuca J, Diamond BJ. Aneurysm of the anterior communicating artery: a review of neuroanatomical and neuropsychological sequelae. J Clin Exp Neuropsychol 1995;17:100-21

3. Fujii T. The basal forebrain and episodic memory. In: Dere E, Nadel L, Easton A, et al, eds. Handbook of Episodic Memory. Amsterdam: Elsevier Science; 2008:343-58

4. Lim C, Alexander MP. Stroke and episodic memory disorders. Neuropsychologia 2009;47:3045-58

5. Proust F, Martinaud O, Gerardin E, et al. Quality of life and brain damage after microsurgical clip occlusion or endovascular coil embolization for ruptured anterior communicating artery aneurysms: neuropsychological assessment. J Neurosurg 2009;110:19-29

6. Markowitsch HJ, Staniloiu A. Amnesic disorders. Lancet 2012;380:1429-40

7. Crowell RM, Morawetz RB. The anterior communicating artery has significant branches. Stroke 1977;8:272-73

8. Sekhar LN, Natarajan SK, Britz GW, et al. Microsurgical management of anterior communicating artery aneurysms. Neurosurgery 2007;61:273-90, discussion 290-92

9. Hernesniemi J, Dashti R, Lehecka M, et al. Microneurosurgical management of anterior communicating artery aneurysms. Surg Neurol 2008;70:8-28, discussion 29

10. Gade A. Amnesia after operations on aneurysms of the anterior communicating artery. Surg Neurol 1982;18:46-49

11. Phillips S, Sangalang V, Sterns G. Basal forebrain infarction: a clinicopathologic correlation. Arch Neurol 1987;44:1134-38

12. Türe U, Yasargil MG, Krisht AF. The arteries of the corpus callosum: a microsurgical anatomic study. Neurosurgery 1996;39:1075-84, discussion 1084-85

13. Serizawa T, Saeki N, Yamaura A. Microsurgical anatomy and clinical significance of the anterior communicating artery and its perforating branches. Neurosurgery 1997;40:1211-16, discussion 1216-18

14. Guglielmi G. Coil over clip. J Neurosurg 2009;111:410-11, author reply $411-12$

15. Heros RC. Perforator and secondary branch origin: the importance of perforators in aneurysm surgery. World Neurosurg 2013 Mar 14. [Epub ahead of print]

16. Marinkovic S, Milisavljevic M, Marinković Z. Branches of the anterior communicating artery: microsurgical anatomy. Acta Neurochir (Wien) 1990;106:78-85

17. von Cramon DY, Muller U. The septal region and memory. Adv Tech Stand Neurosurg 1998;24:3-40

18. Wechsler D. Wechsler Adult Intelligence Scale, third edition (WAISIII). San Antonio: Psychological Corporation; 1997

19. Wechsler D. Wechsler Memory Scale Revised: Manual. San Antonio: Psychological Corporation; 1987

20. Foster JK. Memory impairment. In: Foster JK, ed. Memory: A Very Short Introduction. New York: Oxford University Press; 2009:84-100

21. Hennig J, Scheffler K. Easy improvement of signal-to-noise in RARE-sequences with low refocusing flip angles: rapid acquisition with relaxation enhancement. Magn Reson Med 2000;44:983-85

22. Mai JK, Paxinos G, Voss T. Atlas of the Human Brain. New York: Academic Press; 2007

23. Feekes JA, Hsu SW, Chaloupka JC, et al. Tertiary microvascular territories define lacunar infarcts in the basal ganglia. Ann Neurol 2005;58:18-30

24. Takahashi S. Intracranial arterial system: basal perforating arteries. 
In: Takahashi S, ed. Neurovascular Imaging: MRI and Microangiography. London: Springer-Verlag; 2010:53-130

25. Yaşargil MG, Smith RD, Young PH, et al. Anterior cerebral artery complex. In: Yaşargil MG, ed. Microneurosurgery. Stuttgart: Georg Thieme Verlag; 1984:92-128

26. Takahashi S, Goto K, Fukasawa H, et al. Computed tomography of cerebral infarction along the distribution of the basal perforating arteries. Part I. Striate arterial group. Radiology 1985;155:107-18

27. Takahashi S, Suzuki M, Matsumoto K, et al. Extent and location of cerebral infarcts on multiplanar MR images: correlation with distribution of perforating arteries on cerebral angiograms and on cadaveric microangiograms. AJR Am J Roentgenol 1994;163:1215-22

28. Tsivilis D, Vann SD, Denby C, et al. A disproportionate role for the fornix and mammillary bodies in recall versus recognition memory. Nat Neurosci 2008; 11:834-42

29. Park SA, Hahn JH, Kim JI, et al. Memory deficits after bilateral anterior fornix infarction. Neurology 2000;54:1379-82

30. Moussouttas M, Giacino J, Papamitsakis N. Amnestic syndrome of the subcallosal artery: a novel infarct syndrome. Cerebrovasc Dis 2005;19:410-14

31. Renou P, Ducreux D, Batouche F, et al. Pure and acute Korsakoff syndrome due to a bilateral anterior fornix infarction: a diffusion tensor tractography study. Arch Neurol 2008;65:1252-53

32. Adamovich BL, Gualberto G, Roberts T, et al. Teaching neuroimages: amnesia due to fornix infarction. Neurology 2009;73:e86

33. Hattingen E, Rathert J, Raabe A, et al. Diffusion tensor tracking of fornix infarction. J Neurol Neurosurg Psychiatry 2007;78:655-56

34. Mosimann PJ, Saint-Maurice JP, Lenck S, et al. Fornix infarction and Korsakoff dementia after coiling of a large anterior communicating artery aneurysm. Neurology: Clinical Practice 2012;2:260-62
35. Hong JH, Choi BY, Chang $\mathrm{CH}$, et al. Injuries of the cingulum and fornix after rupture of an anterior communicating artery aneurysm: a diffusion tensor tractography study. Neurosurgery 2012;70:819-23

36. Böttger S, Prosiegel M, Steiger HJ, et al. Neurobehavioural disturbances, rehabilitation outcome, and lesion site in patients after rupture and repair of anterior communicating artery aneurysm. J Neurol Neurosurg Psychiatry 1998;65:93-102

37. Fujii T, Suzuki M, Suzuki K, et al. Normal memory and no confabulation after extensive damage to the orbitofrontal cortex. J Neurol Neurosurg Psychiatry 2005;76:1309-10

38. Fujii T. Perforating branches of the anterior communicating artery: anatomy and infarction. In: Taakahashi S, ed. Neurovascular Imaging: MRI and Microangiography. London: Springer-Verlag; 2010:189-96

39. Claassen J, Bernardini GL, Kreiter K, et al. Effect of cisternal and ventricular blood on risk of delayed cerebral ischemia after subarachnoid hemorrhage: the Fisher scale revisited. Stroke 2001; 32:2012-20

40. Dick JP, Guiloff RJ, Stewart A, et al. Mini-mental state examination in neurological patients. J Neurol Neurosurg Psychiatry 1984;47: 496-99

41. Budson AE, Price BH. Memory dysfunction. N Engl J Med 2005;352:692-99

42. Shah A, Jhawar SS, Goel A. Analysis of the anatomy of the Papez circuit and adjoining limbic system by fiber dissection techniques. J Clin Neurosci 2012;19:289-98

43. Duvernoy HM. Structures, functions, and connections. In: Duvernoy HM, Cattin F, Risold PY. The Human Hippocampus: Functional Anatomy, Vascularization and Serial Sections with MRI. 2nd ed. Berlin: Springer-Verlag; 1998:5-38 